\title{
Behçet's Disease Initially Presenting with Meningitis and Sudden Hearing Loss
}

\author{
Tomomi Kikuchi ${ }^{1}$, Isamu Yokoe ${ }^{1}$, Atsushi Masuyama ${ }^{1}$, Keiichiro Maniwa ${ }^{1}$, Shinji Tsuruta ${ }^{1}$, \\ Yuki Hatanaka ${ }^{2}$ and Hitomi Haraoka ${ }^{1}$
}

\begin{abstract}
We report a patient with neuro Behçet's disease (BD) initially presenting with meningitis and severe hearing loss. A 51-year-old man with no noteworthy past history was hospitalized for evaluation of non-pulsating headache and high fever. Lumbar puncture on admission only showed slight pleocytosis $(6 / \mu \mathrm{L}$, monomorphonuclear cells predominantly) but no evidence of meningitis. However, after admission, he continued to have a fever of over 38 degrees, and he developed painful oral aphthous ulcer and pseudofolliculitis on the upper limbs and trunk. Prior to admission he had often experienced oral ulceration and had bought commercially available mouthwash for prophylaxis. Subsequently, genital ulceration appeared. A small ulcer was observed at the blood collection site, leading to the diagnosis of BD. On the seventh hospital day, the patient developed sudden hearing loss, which was diagnosed as severe sensorineural hearing loss. Lumbar puncture was performed again. Cerebrospinal fluid (CSF) analysis showed mild pleocytosis (60/ $\mu \mathrm{L}$, predominantly monomorphonuclear cells), with high CSF IL-6 levels. Neither edematous change nor atrophy of the brainstem was noted and there were no other abnormal findings on the brain MRI/MRA. Auditory brainstem response was normal, suggesting that the patient had developed hearing loss due to peripheral neuropathy. We speculate that the hearing loss was likely due to vasculitis associated with BD. This case is considered to be a rare case of Behçet's disease caused by severe hearing loss and meningitis.
\end{abstract}

Key words: Behçet's disease, sudden hearing loss, meningitis

(Inter Med 49: 483-486, 2010)

(DOI: 10.2169/internalmedicine.49.2742)

\section{Introduction}

Behçet's disease (BD) was first defined by Hulusi Behçet in 1937. It is a refractory multisystem disorder mainly presenting with recurrent oral aphthous ulcers, uveitis, skin lesions, and genital ulcers, characterized by recurrent acute attacks of inflammation. BD patients having intestinal, vascular, and neurological involvement are categorized as special types according to Japanese classification criteria of Behçet's disease. Central nervous system lesions of BD are classified into two types: acute lesions such as meningitis and brainstem encephalitis, and chronic progressive lesions causing neurological (cerebellar and pyramidal) and psychiatric (dementia) symptoms.

We report a patient who visited our hospital with the chief complaint of headache and was found to have aseptic meningitis on admission, and subsequently developed BD which initially presented with pseudofolliculitis, oral aphthous ulcers, genital ulcer and sudden hearing loss.

\section{Case Report}

The patient was a 51-year old man with the chief complaints of headache and fever. He was an occasional drinker with no history of smoking, but with a history of childhood asthma and atopic dermatitis. He developed a fever of $38^{\circ} \mathrm{C}$ and diffuse, squeezing, and non-throbbing headache in midApril 2009, and subsequently vomited twice. Since the symptoms tended to worsen, he was transported to our emergency room, and was admitted for further examination of headache and fever. He was $170 \mathrm{~cm}$ tall and weighed

${ }^{1}$ Division of Rheumatology, Itabashi Chuo Medical Center, Tokyo and ${ }^{2}$ Department of Neurology, Teikyo University School of Medicine, Tokyo Received for publication August 5, 2009; Accepted for publication October 29, 2009 Correspondence to Dr. Hitomi Haraoka, pero1995@gmail.com 
$64.3 \mathrm{~kg}$. His body temperature was $38.6^{\circ} \mathrm{C}$, with blood pressure of $120 / 60 \mathrm{mmHg}$, and pulse of 74 beats/min. Conjunctivae were not anemic or icteric. No decrease in visual acuity was observed. The oral cavity showed no reddening of mucosa or white furring of the tongue. His tonsils were not swollen. There was no cervical lymphadenopathy. Respiratory sounds were normal, and no heart murmurs were audible. The abdomen was flat, soft, pain-free, and non-tender, with normal bowel sounds. Neurological examination showed clear consciousness and isocoric pupils $(3 \mathrm{~mm}$ in diameter) reacting promptly to light with no apparent laterality. There was no evidence of cranial nerve abnormalities, or signs of meningeal irritation such as neck stiffness, Kernig's sign and Brudzinski's sign. Blood tests showed an elevated WBC of $11,000 / \mu \mathrm{L}$ (with a differential count of $78.2 \%$ neutrophils, $0.1 \%$ eosinophils, $0.2 \%$ basophils, $9.4 \%$ monocytes, $12.1 \%$ lymphocytes) and slightly elevated erythrocyte sedimentation rate of $22 \mathrm{~mm} / \mathrm{h}$. The $\mathrm{C}$ reactive protein (CRP) and ferritin levels were high, at $11.42 \mathrm{mg} / \mathrm{dL}$ and $512 \mathrm{ng} /$ $\mathrm{mL}$, respectively. Protein fractionation revealed an acute inflammation-type response: $19.5 \%$ albumin, $9.7 \% \quad \alpha 1$ globulin, 38.3\% $\alpha 2$-globulin, 9.6\% $\beta$-globulin and $22.9 \% \gamma-$ globulin. Therefore, we began the treatment with cefazolin 2 $\mathrm{mg} /$ day and loxoprofen $180 \mathrm{mg} /$ day (NSAIDs) from the day of admission. Immunological tests were negative for antinuclear antibody, p-antineutrophilic cytoplasmic antibody (ANCA), c-ANCA, rheumatoid factor (RF), rheumatoid arthritis particle agglutination (RAPA), and anti-cyclic citrullinated peptide (CCP) antibody. Complement levels were within normal limits. Tests for various infections were negative (HBs antigen, anti-HCV antibody, qualitative RPR, qualitative TPHA, HIV, rapid antigen detection test for group A $\beta$-hemolytic streptococcus, ASO/ASK, anti-CMVIgM/IgG antibody, influenza A/B virus, herpes simplex IgGIgM, herpes simplex DNA, and Chlamydia pneumoniae). Blood, urine, throat swab, and CSF cultures were negative. Tests for thyroid function and tumor markers (CEA, CA199, PSA, and soluble IL-2R) showed no abnormalities.

Brain CT revealed no specific findings, and CSF analysis on admission revealed a clear, colorless fluid with a slightly elevated cell count of $6 / \mu \mathrm{L}(\mathrm{M} / \mathrm{L}$ ratio, 1:2) but excluded bacterial meningitis. Remittent fever of over $38^{\circ} \mathrm{C}$ persisted after admission. Since blood tests showed an inflammatory response, we suspected some kind of infection, malignant tumor, or collagen disease and continued further evaluations. Blood, urine, sputum, and CSF cultures were all negative, and echocardiography showed no abnormalities. Therefore, we decided on the discontinuation of cefazolin on the fifth hospital day. Lumbar spine MRI revealed no apparent abnormalities, and thoracoabdominal CT scans showed no areas suggestive of infection. On the third hospital day, the patient developed painful aphtha. Subsequently, he developed multiple areas of pseudofolliculitis of approximately 2 $\mathrm{mm}$ in diameter on the upper limbs and trunk, and later, genital ulcers. A small ulcer was observed in the right groin at the site of blood collection for blood gas analysis. It also



\begin{tabular}{|c|c|c|c|c|}
\hline \multirow{2}{*}{} & \multicolumn{2}{|c|}{ AVE.4 } & \multicolumn{2}{c|}{ AVE.6 } \\
\cline { 2 - 5 } & AIR & BONE & AIR & BONE \\
\hline R & $58.8 \mathrm{~dB}$ & - & $60.0 \mathrm{~dB}$ & - \\
\hline L & $27.5 \mathrm{~dB}$ & - & $24.2 \mathrm{~dB}$ & - \\
\hline
\end{tabular}

Figure 1. Mean right hearing level was $59 \mathrm{~dB}$ by air conduction, with laterality of hearing. At time of pure tone audiometry performance, the patient became ill and bone conduction test could not be performed.

became clear that he had experienced painful aphtha many times in the past and mosquito bites had often left weals. On the seventh hospital day, he experienced sudden onset of tinnitus and hearing loss, and was diagnosed with severe sensorineural hearing loss on otolaryngologic consultation next day. In Fig. 1, the mean right hearing level was $59 \mathrm{~dB}$ by air conduction, indicating severe sensorineural hearing loss. At the time of pure tone audiometry performance, the patient became ill and bone conduction test could not be performed. However, a diagnosis of otitis media was unlikely since no tympanic membrane finding was observed upon air conduction test, with laterality of hearing. Still there were no signs of meningeal irritation, and we performed a second lumbar puncture which showed clear, colorless fluid with a further elevated cell count of $60 / \mu \mathrm{L}(\mathrm{M} / \mathrm{L}$ ratio, 1:4), protein level of $53 \mathrm{mg} / \mathrm{dL}(15-40)$, and glucose level of $62 \mathrm{mg} / \mathrm{dL}$. On subsequent examination, oligoclonal band and myelin basic protein were negative, and anti-virus antibody titers in paired sera showed no significant change. Based on mucocutaneous symptoms such as recurrent painful aphthous ulcers, pseudofolliculitis, genital ulcers and ulcerations at the venopuncture site, we made the diagnosis of $\mathrm{BD}$, although uveitis was not found. The elevated CSF IL-6 level of $89 \mathrm{pg} / \mathrm{mL}$, measured by CLEIA (chemiluminescent enzyme immunoassay), suggested aseptic meningitis due to $\mathrm{BD}$, leading to the diagnosis of acute-type neuro-BD. Brain MRI and MRA revealed no edematous changes or atrophy in the brainstem, as observed in acute phase of $\mathrm{BD}$, with no other abnormalities. In addition, auditory brainstem response 
(ABR) was normal. As shown in Fig. 2, stimulation with 70 and $90 \mathrm{~dB}$ produced $\mathrm{ABR}$ waves I through $\mathrm{V}$ with normal latency and amplitude without laterality, showing no obvious abnormalities. Thus, we concluded that there were no brainstem lesions that could cause sudden hearing loss, and considered peripheral neuropathy of BD as the cause of sensorineural hearing loss. The patient was started on prednisolone therapy $(1.0 \mathrm{mg} / \mathrm{kg} /$ day $)$ for meningitis of acute-type neuro-BD and sudden hearing loss. On the following day (8 th hospital day), the fever resolved, and headache, hearing loss and tinnitus improved. Inflammatory response also im-

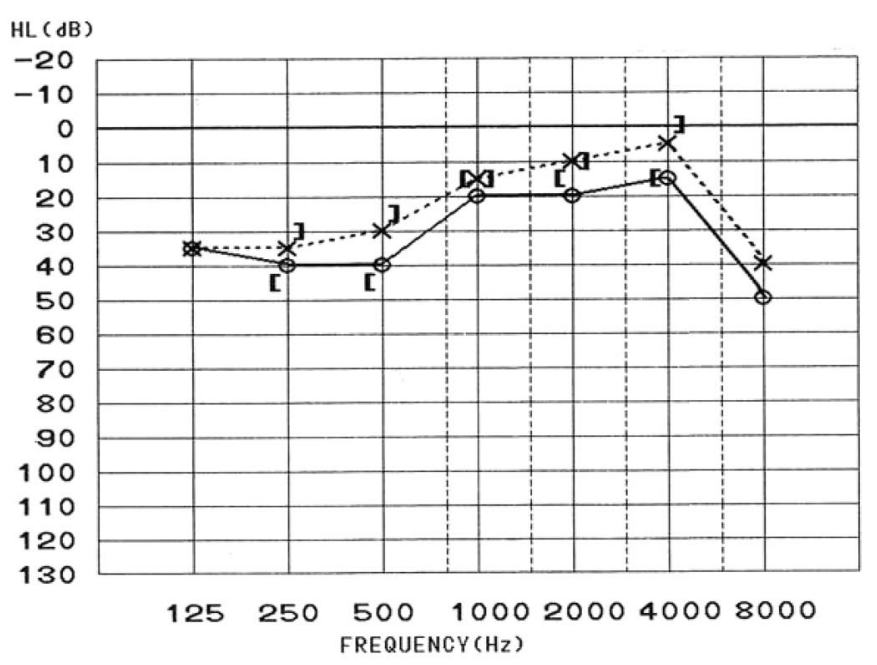

\begin{tabular}{|c|c|c|c|c|}
\hline \multirow{2}{*}{} & \multicolumn{2}{|c|}{ AVE. } & \multicolumn{2}{c|}{ AVE.6 } \\
\cline { 2 - 5 } & AIR & BONE & AIR & BONE \\
\hline$R$ & $25.0 \mathrm{~dB}$ & $22.5 \mathrm{~dB}$ & $22.5 \mathrm{~dB}$ & $20.0 \mathrm{~dB}$ \\
\hline $\mathrm{L}$ & $17.5 \mathrm{~dB}$ & $16.3 \mathrm{~dB}$ & $14.2 \mathrm{~dB}$ & $12.5 \mathrm{~dB}$ \\
\hline
\end{tabular}

Figure 2. Hearing level had returned to normal. A-B gap(-). proved, and CRP became negative on the 11th hospital day. Audiometric test performed in mid-May (22nd hospital day) showed that hearing level had returned to normal (Fig. 3). Therefore, sensorineural deafness was most suspected from the clinical course. Tinnitus had almost completely disappeared. CSF examination performed again on the 14th hospital day revealed a clear, colorless fluid with normal protein level of $23 \mathrm{mg} / \mathrm{dL}$ and normal cell count; thus prednisolone was reduced to $0.8 \mathrm{mg} / \mathrm{kg} / \mathrm{day}$. Subsequent tests were positive for HLA-B51. On the 28th hospital day, the CSF IL-6 level was below the detection limit. Thereafter, meningitis did not recur despite prednisolone dose reduction.

\section{Discussion}

Neuro-Behçet's disease is a late-onset disease, and usually becomes manifest 5-10 years after repeated severe attacks of Behçet's disease. It accounts for 5-10\% of all BD patients, with no apparent gender difference. The present patient appeared to have had aseptic meningitis since admission despite only slight CSF pleocytosis. Subsequently, BD manifested itself with painful aphtha, genital ulcers, and, peudofolliculitis, followed by severe sensorineural hearing loss. CSF findings were characteristic of neuro-BD in that the IL6 level was high, the protein level was moderately elevated, cell count was increased, and few neutrophils were observed initially but were later replaced by lymphocytes. Brain MRI on T2-weighted and FLAIR images revealed no abnormalities characteristic of neuro-BD, such as hyperintense lesions, edematous changes, and brainstem atrophy, nor thrombosis of superior sagittal sinus or other dural sinuses, in common BD.

Hearing loss occurs relatively rarely in patients with BD,

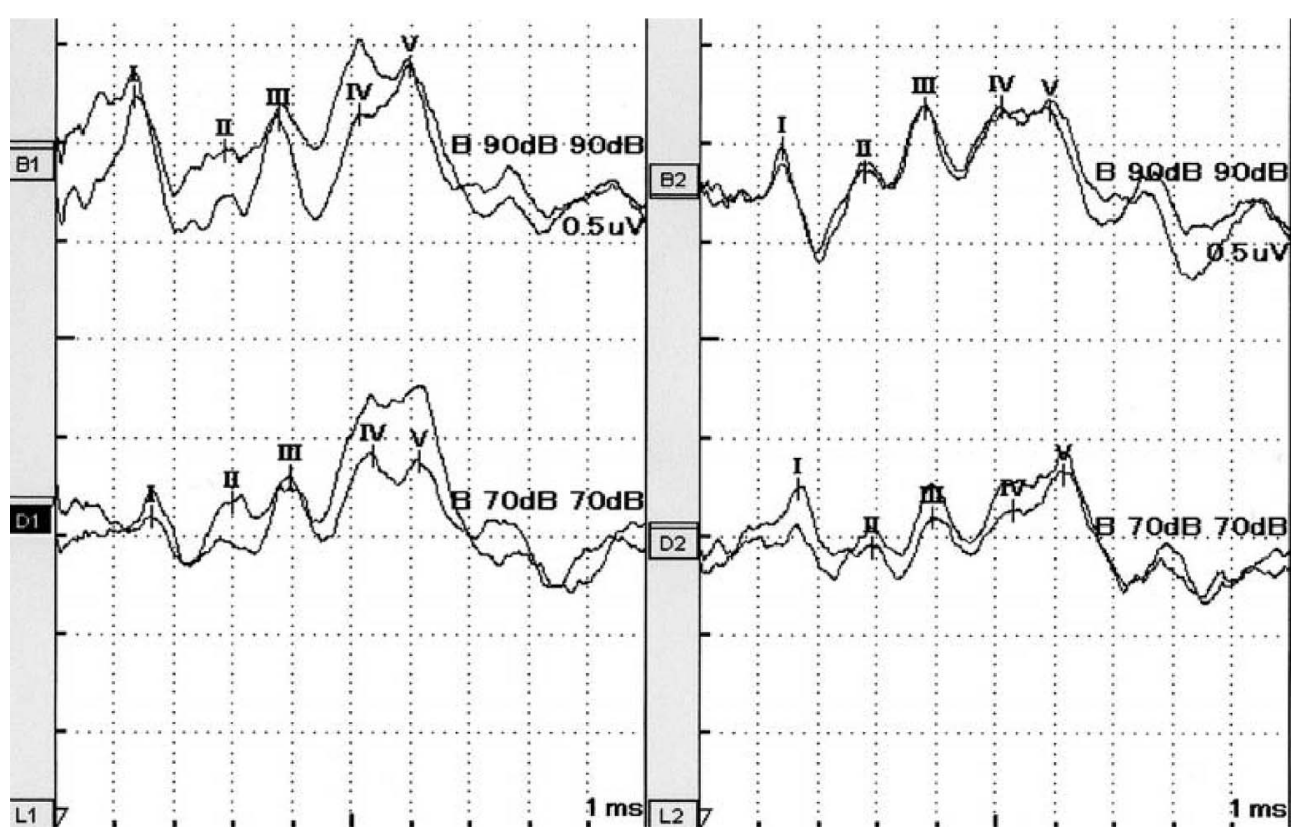

Figure 3. Stimulation with 70 and $90 \mathrm{~dB}$ produced $A B R$ waves $I$ through $V$ with normal latency and amplitude without laterality, showing no obvious abnormalities. 
but latent hearing loss, mainly sensorineural hearing loss, occurs in about $50 \%$ of BD patients by audiometry (1). In particular, HLA-B51-positive patients were reported to have a high risk of hearing loss (2). Many patients with BD presenting with hearing loss were noted to have mild sensorineural hearing loss (3). However, an approximately 60$\mathrm{dB}$ severe sensorineural hearing loss, as in the present case, seems rare.

Causes of sensorineural hearing loss include central and peripheral nerve disorders. In particular, sensorineural hearing loss due to peripheral neuropathy was reported to be very rare (4). In the present case, brain MRI and CT scans revealed no brainstem lesions, and ABR was normal, strongly suggesting that hearing loss was not due to central nerve disorder, but was due to peripheral inner ear disorder. Studies have reported that peripheral hearing loss in BD is due to inner ear vasculitis associated with BD (5-7). This was probably the case in the present patient. Inner ear damage due to vasculitis may also be observed in connective tissue diseases such as systemic lupus erythematosus, Wegener's granulomatosis, and polyarteritis nodosa (7-9). It is believed that disseminated vasculitis due to immune complex deposition affects the cochlea. A common underlying histopathological lesions in Behcet's disease is vasculitis with perivascular infiltration by mononuclear leukocytosis $(9,10)$. Therefore, diffuse pathological features may also involve the inner ear.

In the present case, since the CSF IL-6 level was high, corticosteroid therapy was started for treatment of meningitis of acute-type neuro-BD. Thereafter, the clinical symptoms of headache, tinnitus, and hearing loss were improved. In addition, subsequent CSF examination showed that the IL-6 level was below the detection limit. Corticosteroid was successfully reduced, and hearing returned to normal. If a diagnosis of hearing loss in $\mathrm{BD}$ is delayed, the hearing recovery may be poor (12), and concomitant use of immunosuppressive agents is necessary in some patients (13), underscoring the importance of early diagnosis and treatment.

Some patients with acute-type neuro-BD do not experience fevers or headaches, and finally have a mild stroke attack, requiring no corticosteroid therapy. However, other patients may present with severe symptoms of a neurological disorder. Thus, acute-type neuro-BD presents various clinical states. However, search of the English language medical literature revealed no case reports of acute-type neuro-BD initially presenting with meningitis followed by peripheral hearing loss, making the present case unique. It is important to diagnose acute-type neuro-BD early in every case.

\section{References}

1. Andreoli C, Savastano M. Audiologic pathology in Behçet syndrome. Am J Otol 10: 466-467, 1989.

2. Gemignani G, Berrettini S, Bruschini $P$, et al. Hearing and vestibular disturbances in Behçet's syndrome. Ann Otol Rhinol Laryngol 100: 459-463, 1991.

3. Kulahli I, Balci K, Koseoglu E, Yuce I, Cagli S, Senturk M. Audio-vestibular disturbances in Behcet's patients: report of 62 cases. Hear Res 203: 28-31, 2005.

4. AK E, Harputluoglu U, Oghan F, Baykal B. Behçet's disease and hearing loss. Auris Nasus Larynx 31: 29-33, 2004.

5. Elidan J, Levi H, Cohen E, BenEzra D. Effect of cyclosporine A on the hearing loss in Behçet's disease. Ann Otol Rhinol Laryngol 100: 464-468, 1991.

6. Brama I, Fainaru M. Inner ear involvement in Behcet's disease. Arch Otolaryngol 106: 215-217, 1980.

7. Yoon TH, Paparella MM, Schachern PA. Systemic vasculitis: a temporal bone histopathologic study. Laryngoscope 99: 600-609, 1989.
8. Caldarelli DD, Rejowski JE, Corey JP. Sensorineural hearing loss in lupus erythematosus. Am J Otol 7: 210-213, 1986.

9. Kempf HG. Ear involvement in Wegener's granulomatosis. Clin Otolaryngol Allied Sci 14: 451-456, 1989.

10. France R, Bucherun RN, Wilson MW, Sheldon MB Jr. Relapsing iritis with recurrent ulcers of the mouth and genitalia (Behçet's syndrome); review: with report of additional case. Medicine (Baltimore) 30: 335-355, 1951.

11. Halperin D, Wolf D, Zimhony O. Enteroviruses and sudden deafness. Schattner A, CMAJ 168: 1421-1423, 2003.

12. Soylu L, Aydoğan B, Soylu M, Ozsahinoğlu C. Hearing loss in Behçet's disease. Ann Otol Rhinol Laryngol 104: 864-867, 1995.

13. Narváez J, Valverde-García J, Alegre-Sancho JJ, et al. Sudden cochlear hearing loss in a patient with Behçet's disease. Rev Rhum Engl Ed 65: 63-64, 1998.

(C) 2010 The Japanese Society of Internal Medicine http://www.naika.or.jp/imindex.html 